\title{
Uso de las Tecnologías en el Aprendizaje por Adolescentes desde la Perspectiva de los Padres de Familia. El caso de Educación Secundaria del Sur de Sonora, México
}

\author{
Joel Angulo-Armenta*, Jesús Tánori-Quintana, Sonia V. Mortis-Lozoya y Lupita A. Angulo-Arellanes \\ Instituto Tecnológico de Sonora, Departamento de Educación, Cd. Obregón, Sonora, México. \\ (e-mail: joel.angulo@itson.edu.mx; jesus.tanori@itson.edu.mx; sonia.mortis@itson.edu.mx; \\ lupita.alexandra96@gmail.com). \\ * Autor a quien debe ser dirigida la correspondencia
}

Recibido Ene. 30, 2019; Aceptado Mar. 28, 2019; Versión final May. 27, 2019, Publicado Dic. 2019

\begin{abstract}
Resumen
El propósito de este estudio fue determinar la percepción que tienen los padres de familia sobre el uso que sus hijos adolescentes les dan a las tecnologías (computadora e Internet) en el proceso de aprendizaje. En esta investigación de corte cuantitativo, con diseño transeccional no experimental y de alcance correlacional, la muestra fue no probabilística de 379 padres de familia de adolescentes de educación secundaria del sur de Sonora en México. Dentro del contexto general estudiado, los padres percibieron que sus hijos usan la computadora y la Internet para su aprendizaje, y las cuatro principales dimensiones observadas fueron: realización de actividades académicas, socialización, recreación y búsqueda de la información, las cuales se correlacionan de manera significativa. Finalmente, sobre las implicaciones prácticas y educativas del uso de las tecnologías en el aprendizaje, se concluyó que hay una percepción positiva de los padres de familia hacia el uso de éstas en la educación de sus hijos.
\end{abstract}

\section{Use of Technologies in Learning by Adolescents from the Perspective of Parents. The Case of Secondary Education in the South of Sonora, Mexico}

\begin{abstract}
The purpose of this study was to determine the perception of parents about the use that their adolescents give to technologies (computer and Internet) in the process of learning. In this quantitative research, with nonexperimental transactional design and correlational scope, the sample was non-probabilistic of 379 parents of adolescents from secondary education in southern Sonora, Mexico. Within the general context studied, parents perceived that their children use the computer and the Internet for their learning, and the four main dimensions observed were: academic activities, socialization, recreation and information search, which are correlated in a meaningful way. Finally, on the practical and educational implications of the use of technologies on learning, it was concluded that there is a positive perception of parents towards the use of these in the education of their children.
\end{abstract}

Keywords: internet; computers; perception on technologies; learning 


\section{INTRODUCCIÓN}

Actualmente, en el contexto mundial, la incursión y tendencias hacia una cultura informática escolar se ha tornado innovadora, esto obedece a un uso creciente de las Tecnologías de la Información y Comunicación (TIC) en los procesos de enseñanza y aprendizaje (Losada et al., 2012; Kwok y Yang, 2017). La incorporación de las tecnologías en la educación, específicamente en el currículo ha sido constante, permanente y se ha extendido a todos los niveles educativos, situación que precisa que las nuevas generaciones de estudiantes, en especial los adolescentes, tengan una amplia gama de conocimientos y habilidades en el uso de las TIC (García y Rey, 2013; Morales, 2011); por lo anterior, el uso de la computadora, la Internet y los programas tecnológicos educativos por el alumnado, se han convertido en herramientas útiles para realizar actividades escolares, lo que marca una tendencia en el aprendizaje mediado con tecnologías y cambios en el sistema educativo (Krajka y Kleban, 2014).

En este orden de ideas, la integración de las tecnologías en los sistemas de enseñanza ha contribuido a que el profesorado esté implementando estrategias innovadoras en la práctica docente (Fernandez - Batanero; Colmenero - Ruiz, 2016). Consecuentemente, el proceso formativo, entendido como el conjunto de acciones planificadas para promover la interacción entre estudiantes, recursos educativos y profesores, para lograr resultados de aprendizaje, se ha perfilado hacia una educación basada en la innovación pedagógica, las destrezas creativas y la flexibilidad en el uso de recursos tecnológicos como la Internet y computadora, por mencionar algunos. Además, de que los alumnos logren el dominio paulatino de la competencia tecnológica y mejoren el desempeño para desarrollar sus actividades escolares (Carranza et al., 2017; Hernández et al., 2014; Kumar y Ben, 2016; Mantilla et al., 2014). En consecuencia, la familia ha adquirido un papel preponderante en este trinomio escuela, proceso formativo y uso de las tecnologías; por lo que es de esperarse que los padres de familia o padres (entendido como el papá y la mamá, o representantes) se sigan involucrando más en las actividades escolares de sus hijos adolescentes (para los propósitos de este estudio, las hijas aparecen representadas en el concepto de hijos) facilitando apoyos como son recursos económicos, recursos tecnológicos, y el acompañamiento en el proceso de aprendizaje.

Epstein (2011) asume que la participación que los padres de familia durante el proceso formativo de los hijos, contribuye en los logros académicos y otras actividades relacionadas con la gestión al interior de la institución educativa. En este sentido, la superación o fracaso en el aprendizaje de los adolescentes es derivado de un trabajo colegiado donde interviene de manera directa la familia, ya que es un hecho que los grandes aprendizajes inician en el hogar, a mayor intervención de los padres de familia en la educación de los hijos, el éxito se vislumbra de manera exitosa en su formación para la vida (Angulo et al., 2015). Valdés y Urías (2011) aseguran que cuando un adolescente muestra una actitud positiva hacia el estudio, realiza las tareas adecuadamente y alcanza un óptimo rendimiento académico, esto se asocia necesariamente con los padres de familia; es decir, la participación de los padres en la educación de sus hijos ofrece resultados tangibles. No obstante, sus hijos (Perales y Escobedo, 2016). Cabe mencionar que la intervención y colaboración de los padres en el éxito escolar de los adolescentes, es entendida como las tareas o actividades que colaboran con el centro escolar en la educación de sus hijos (Lewis, 1992).

La participación de la familia debe ser a través de una comunicación oportuna, fácil y útil de entender debido a que brinda oportunidades para intercambiar información con los hijos (Mutch y Collins, 2012). Cuando los padres de familia participan en la formación de sus hijos, esto se asocia a una condición y conducta favorable en la institución por parte del alumno, observándose mayores logros en la lectura, trabajos de mayor calidad y mejor rendimiento académico en general. Asimismo, Epstein y Sheldon (2006) aseguran que la interacción efectiva entre familia - comunidad - escuela hacen que una institución educativa sea exitosa, de hecho, no se podría concebir hoy en día el éxito de los estudiantes sin la participación de estos actores educativos. Las escuelas que presentan mejores resultados suelen ser aquellas donde existe una interacción positiva entre la familia y la institución educativa. En México, la participación de los padres de familia en la educación se inició desde que se reconocieron los cambios planteados el Sistema Educativo Nacional, en el transcurso de los siglos XVIII y XIX, bajo estos cambios se reestructuró la función social de la escuela y su vínculo con los padres de familia; con el paso del tiempo se formalizó la participación de los padres de familia, primero mediante la creación de la Unión de Padres de Familia y después la Asociación Nacional de Padres de Familia. Del mismo modo, existen algunos modelos que se caracterizan por enfocar la participación de los padres en la formación de sus hijos (Epstein, 2011; Martiniello, 1999) como un fenómeno que puede darse en diferentes niveles y dimensiones.

A continuación, se presentan algunos de los más importantes y se señala la forma como cada uno de los modelos conceptualiza la contribución de la familia (padre y madre). Primeramente, Martiniello (1999) expone la Taxonomía de participación de padres, donde se propone analizar la participación de los padres de familia desde las siguientes cuatro dimensiones: (1) Crianza: se refiere al desempeño de las funciones propias de padres y madres, creando las condiciones económicas y psicológicas que permiten al niño asistir a la escuela; 
(2) Maestros: son acciones que desarrollan los padres y madres para continuar y reforzar el proceso de aprendizaje del aula en la casa; (3) Agentes de apoyo: se refiere a las contribuciones que los padres y madres hacen a las escuelas para mejorar la provisión de los servicios (dinero, tiempo, trabajo y materiales); y (4) Agentes con poder de decisión: los padres y madres desempeñan roles de toma de decisión que afectan las políticas de la escuela y sus operaciones (participación de padres de familia en consejos escolares consultivos y directivos, o en programas de selección de escuelas/vales escolares).

En la figura 1, se puede observar la propuesta de Epstein (2002) quien establece seis niveles de involucramiento de los padres de familia en la formación educativa de sus hijos en donde la finalidad es disminuir el distanciamiento entre la escuela y familia. Los niveles son: (1) Escuela para padres (se motiva a la familia para exista una ambiente que apoye los estudios de los niños); (2) Comunicación (se diseñan formas efectivas de comunicación entre familia y escuela); (3) Voluntariado (se hacen actividades donde intervienen los padres de familia en la escuela); (4) Aprendizaje en casa (el centro escolar ofrece ideas a la familia para ayudar a los hijos en sus actividades escolares); (5) Toma de decisiones (los padres de familia son involucrados en la toma de decisiones dentro de la escuela; y (6) Colaboración con la comunidad, (identificar las necesidades de la comunidad para fortalecer los programas escolares, las prácticas familiares y el aprendizaje de los alumnos).

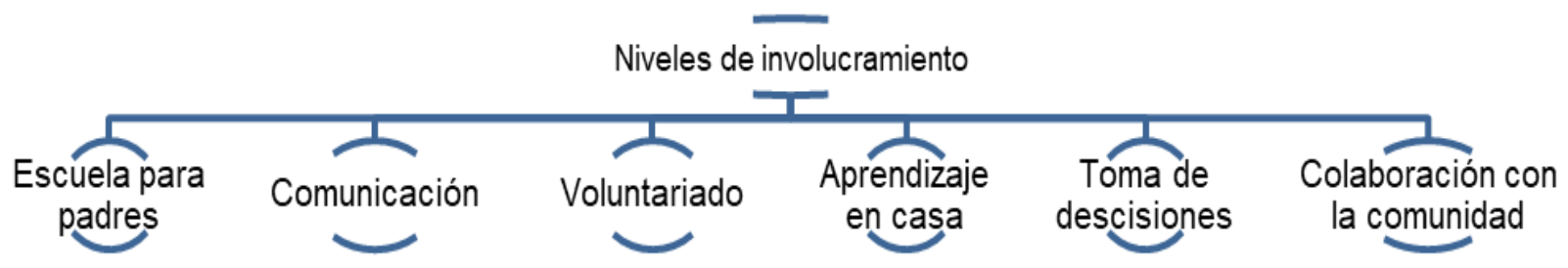

Fig. 1: Niveles de involucramiento de los padres de familia en la educación

(Tomado, con autorización, de Angulo, et al., 2015).

En la actualidad, la tecnología se ha vuelto esencial en la vida de los adolescentes, por ejemplo, la televisión, la Internet, los videojuegos, los dispositivos móviles y muchos otros, ofrecen innumerables oportunidades y beneficios en la educación. Ante esto las generaciones actuales (los llamados Millennials o nativos digitales) están cambiando la forma de aprender (Noguera, 2015). No obstante, el abuso de estos aparatos electrónicos es usualmente realizado por los adolescentes los cuales consumen la mayor parte de su tiempo en utilizar estos medios para socializar y actividades lúdicas. Al presentarse estos casos, los padres y las madres deben ser los responsables de influir en sus hijos para el buen uso de los recursos tecnológicos.

Referente al contexto del problema de estudio, en México la Asociación Mexicana de Internet (Amipci, 2019) reporta que hoy en día hay 79.1 millones de usuarios de la Internet y que el $18 \%$ lo constituyen a adolescentes entre 12 a 17 años. Asimismo, se observa que los horarios de mayor tráfico en la Internet, es en medio día (12 a 14 horas) y a media tarde (16 a 19 horas); además, el $67 \%$ de los internautas se percibe que está conectado las 24 horas. El $84 \%$ se conecta en el hogar, el $67 \%$ desde cualquier lugar, el $8 \%$ desde la escuela, el $42 \%$ de internautas utilizan la Internet sobre todo para el ocio, ver películas y escuchar música (56\%); formación, cursos en línea (46\%); conocer gente, hacer amigos, conseguir pareja (42\%); entre otros. Ante este panorama, se observa que hay un uso desmedido de las TIC para esparcimiento y para la educación de los adolescentes, de aquí se desprende la necesidad de los padres de familia de involucrarse más en el aprendizaje de sus hijos ya que son el principal referente de sus conductas en cuanto a los valores y usos que le dan a las TIC en el aprendizaje. Contrariamente a lo expresado, Matías, Silva y Viana (2018) aseguran que las familias no constituyen un grupo homogéneo al interior de la misma, esto es, la integración de las TIC puede tornarse complicado debido a que se relaciona con la brecha generacional y el género tanto en los padres como en los adolescentes.

Gurpegui (2010) plantea algunas sugerencias sobre el uso de las TIC dentro de la familia para propiciar el aprendizaje: (1) se precisa fomentar la interacción y comunicación a través de la Internet que ayuden a los hijos a aprender; (2) las tecnologías son recursos de aprendizaje y comunicación que pueden ayudar a desarrollar habilidades cognitivas, la creatividad y motivar a los estudiantes a realizar actividades escolares; y (3) se pueden realizar consultas, exámenes en línea, gestiones escolares, contactar a docentes, hacer proyectos académicos, tener información del centro escolar y recibir orientaciones docentes, entre otras. Debido a que es el hogar donde se establece el mayor tiempo de interacción entre la familia, el adolescente y las TIC; los padres deben definir su participación en las tareas de los hijos, desarrollando en ellos un sentido de responsabilidad, independencia y comunicación (Cunha, et al., 2015), no obstante, esto no garantiza que la comunicación sea efectiva y prevalezca, lo que puede generar limitaciones en el apoyo por parte de los padres de familia. Puesto que este fenómeno donde la familia participa en el aprendizaje de sus hijos mediado por las TIC es generalizado y existe limitada documentación al respecto, surgió la necesidad de realizar un 
estudio en escuelas de educación básica obligatoria, en un municipio del sur del Estado de Sonora, que permitió identificar la percepción que tienen los padres de familia sobre el uso que sus hijos de nivel secundaria le dan a las TIC en su proceso formativo en factores como: Realización de actividades académicas, Socialización, Recreativos, y Búsqueda de la información. En México, la educación secundaria es el último trayecto de la enseñanza básica obligatoria la cual se estructura por el nivel preescolar de 3 a 5 años de edad, nivel primaria de 6 a 11 años de edad, y nivel secundaria de 12 a 15 años de edad).

Por lo anterior, el objetivo general de este estudio fue determinar la percepción que tienen los padres de familia sobre el uso que sus hijos adolescentes le dan a las TIC en su proceso formativo. Los objetivos específicos se plantearon con el siguiente alcance: (1) Determinar el nivel de frecuencia que percibe el padre de familia respecto al apoyo que brinda la computadora y la Internet en el aprendizaje de sus hijos adolescentes; y (2) Establecer relaciones entre los factores de (a) Realización de actividades académicas, (b) Socialización, (c) Recreativos, y (d) Búsqueda de la Información que percibe el padre de familia ante el uso que sus hijos adolescentes hacen de las TIC. Esto hace suponer que existe un contexto el cual propicia el uso con la tecnología, tal como: tener computadora en casa y acceso a la Internet.

\section{MATERIALES Y MÉTODOS}

La metodología se presenta en tres subsecciones: en la primera se describe el tipo de estudio y participantes; en la segunda se explica el instrumento, sus características y un resumen del proceso de validación; y en la tercera de detalló el procedimiento.

El tipo de estudio fue de corte cuantitativo, con un diseño transversal no experimental de alcance correlacional. La población estuvo constituida por padres de familia de estudiantes adolescentes inscritos en los grados de primero a tercero en escuelas públicas de educación obligatoria del sur de Sonora en México, las edades oscilaron entre los 12 y los 16 años, de donde fueron seleccionados 379 padres de familia (papás, mamás o representantes que corresponden a igual número de adolescentes) de forma no aleatoria que respondieron el instrumento, los datos se recolectaron en el verano del 2018. Las edades de los padres de familia en promedio fueron de 38 años $(D S=7.18)$ con una edad mínima de 23 y máxima de 73 años. En cuanto al nivel educativo de los padres el 19.8\% (75) posee licenciatura, el $29.3 \%$ (111) bachillerato, el $43.3 \%$ (164) secundaria, el $7.1 \%$ (27) nivel primaria, y por último un .5\% (2) padres de familia no concluyeron ningún nivel de estudio.

Referente al instrumento, se administró la escala "Percepción de padres sobre sus hijos estudiantes ante el uso de las tecnologías" (Urías, Angulo, Valdés y Torres, 2015), con la finalidad de recabar información sobre la percepción que tienen en el uso de las tecnologías por parte de sus hijos adolescentes en el proceso formativo. Se constituyó en una primera sección con 14 preguntas de datos generales y la segunda constó de 28 ítems que se respondieron con una escala tipo Likert que osciló desde 7 (Siempre) hasta 0 (No tengo información). Se compuso de una tabla de especificaciones con cuatro factores derivados de la teoría, que se explican a continuación: (a) Realización de actividades académicas (recursos tecnológicos que apoyan para facilitar el aprendizaje escolar); (b) Socialización (comunicarse e interactuar por medio de la tecnología con otra persona); (c) Recreativos (utilidad de diversas aplicaciones tecnológicas de interés); y (d) Búsqueda de información con fines académicos (habilidades en el uso de las tecnologías empleadas en tareas y actividades). Se realizó un análisis factorial exploratorio utilizando el método de factorización de ejes principales donde se obtuvo una medida de adecuación muestral de Kaiser-Meyer-Olkin adecuada $(\mathrm{KMO}=$ .921), y una prueba de esfericidad de Bartlett significativa $\left(X^{2}=6350.98, p<.000\right)$, lo cual confirma la adecuación de los datos para el análisis factorial. Se obtuvo una solución integrada por cuatro factores que explican el $53 \%$ de la varianza y por último se determinó la consistencia interna, mediante el coeficiente de confiabilidad alfa de Cronbach para el instrumento final que fue de .94 siendo este excelente.

Finalmente, para la recolección de los datos se efectuaron las fases o actividades que se describen a continuación: $\left(1^{\circ}\right)$ se solicitó autorización a los directores de las instituciones educativas para convocar a reuniones de padres de familia; $\left(2^{\circ}\right)$ en cada reunión se pidió permiso y la colaboración voluntaria a los padres de familia quienes otorgaron su consentimiento informado y se les garantizó confidencialidad de la información por el equipo de aplicadores; $\left(3^{\circ}\right)$ se enviaron instrumentos, a través de sus hijos, a los padres de familia que no asistieron a las reuniones (previo acuerdo con los profesores de grupo que apoyaron en recolectar los instrumentos contestados); y $\left(4^{\circ}\right)$ con los instrumentos recolectados, se elaboró la base de datos y se analizaron los datos a través de estadística descriptiva e inferencial.

\section{RESULTADOS Y DISCUSIÓN}

Los resultados se explican según los objetivos específicos del estudio, se procuró hacer un detalle de las observaciones encontradas en el estudio. 
Objetivo 1. Determinar el nivel de frecuencia. Para determinar si la computadora y la Internet apoyan el aprendizaje de sus hijos en el hogar (aprendizaje en casa), se calculó la frecuencia ante estos dos datos generales, donde se encontró que en ambos casos regularmente lo toman como apoyo según la percepción de los padres de familia, tal y como se muestra en la tabla 1.

Tabla 1: La computadora y el Internet como apoyo en el aprendizaje.

\begin{tabular}{|c|c|c|c|c|}
\hline & \multicolumn{2}{|c|}{ Computadora } & \multicolumn{2}{c|}{ Internet } \\
\hline Categoría & Frecuencia & Porcentaje & Frecuencia & Porcentaje \\
\hline Poco & 19 & 5.0 & 24 & 6.3 \\
\hline Regularmente & 261 & 68.9 & 270 & 71.2 \\
\hline Siempre & 99 & 26.1 & 85 & 22.5 \\
\hline Total & 379 & 100 & 379 & 100 \\
\hline
\end{tabular}

Objetivo 2. Establecer relaciones entre los factores. Para establecer relaciones entre los factores Realización de actividades académicas, Socialización, Recreativos y Búsqueda de la información, se realizó una correlación bivariante con el coeficiente de Pearson, así se observa en la tabla 2. Se observó que existen correlaciones medianas a considerables, positivas y significativas entre los factores que se encargan de medir la percepción del padre de familia sobre el uso que tienen sus hijos de las TIC en las dimensiones expuestas.

Tabla 2: r de Pearson entre los factores de la percepción de padres sobre sus hijos estudiantes ante el uso de las TIC.

\begin{tabular}{|l|c|c|c|c|c|c|}
\hline \multicolumn{1}{|c|}{ Dimensiones } & $M$ & $D . E$. & 1 & 2 & 3 & 4 \\
\hline Realización de actividades académicas & 2.43 & 1.17 & & & & \\
\hline Socialización & 3.94 & 2.03 & $.536^{\star *}$ & & & \\
\hline Actividades recreativas & 2.80 & 1.19 & $.581^{* *}$ & $.552^{* \star}$ & & \\
\hline Búsqueda de la información con fines académicos & 4.24 & 1.78 & $.583^{*}$ & $.651^{\star \star}$ & $.578^{\star *}$ & \\
\hline
\end{tabular}

Para conocer si existen diferencias significativas entre el estado civil de los padres de familia (variables socio demográficas) y las dimensiones del instrumento aplicado (variables sociodemográficas), se hizo una comparación de medias mediante una prueba $t$ de student para muestras independientes (ver tabla 3). Encontrándose que no existen diferencias significativas en cuanto al estado civil de los padres de familia y su percepción ante el uso que sus hijos como estudiantes tienen de las TIC.

Tabla 3: Resultados de la Prueba t de Student Estado Civil y los factores.

\begin{tabular}{|l|c|c|c|c|c|c|c|}
\hline \multicolumn{1}{|c|}{ Dimensiones } & \multicolumn{2}{c|}{$\begin{array}{c}\text { Soltero } \\
(n=93)\end{array}$} & \multicolumn{2}{c|}{$\begin{array}{c}\text { Casado } \\
(n=286)\end{array}$} & $t$ & $g l$ & $p$ \\
\hline & $\mathrm{M}$ & $\mathrm{DS}$ & $\mathrm{M}$ & $\mathrm{DS}$ & & & \\
\hline Realización de actividades académicas & 2.45 & 1.40 & 2.39 & 1.09 & 1.16 & 377 & .246 \\
\hline Socialización & 3.97 & 2.26 & 3.94 & 1.96 & .15 & 377 & .877 \\
\hline Actividades recreativas & 2.82 & 1.41 & 2.79 & 1.11 & .24 & 377 & .810 \\
\hline Búsqueda de la información con fin académico & 4.24 & 2.04 & 4.26 & 1.68 & .11 & 378 & .908 \\
\hline
\end{tabular}

Por otro lado, para conocer si existen diferencias significativas en contar con computadora e Internet en casa de los padres de familia y las dimensiones del instrumento, se realizó una comparación de medias para lo cual se utilizó una prueba $t$ de Student para muestras independientes. En la tabla 4 se observa que existen diferencias significativas en cuanto a tener computadora en casa por parte de los padres de familia y su percepción ante el uso que sus hijos como estudiantes tienen de las TIC. El tamaño del efecto de estas diferencias es bueno puesto que cumple con los criterios mínimos para determinar que esa diferencia entre las medias es verdadera y se sustenta mediante la prueba de Cohen's. 
Tabla 4: Resultados de la Prueba t de Student Contar con computadora en casa.

\begin{tabular}{|l|c|l|c|l|c|c|c|c|}
\hline \multicolumn{1}{|c|}{ Dimensiones } & \multicolumn{2}{|c|}{$\begin{array}{c}\mathrm{Si} \\
(\mathrm{n}=265)\end{array}$} & \multicolumn{2}{c|}{$\begin{array}{c}\mathrm{No} \\
(\mathrm{n}=114)\end{array}$} & $\mathrm{t}$ & $\mathrm{gl}$ & $\mathrm{p}$ & $d$ \\
\hline & $\mathrm{M}$ & $\mathrm{DS}$ & $\mathrm{M}$ & $\mathrm{DS}$ & & & & \\
\hline Realización de actividades académicas & 2.63 & 1.15 & 1.85 & .97 & -6.14 & 377 & .000 & .30 \\
\hline Socialización & 4.43 & 1.91 & 2.82 & 1.87 & -7.58 & 377 & .000 & .36 \\
\hline Actividades recreativas & 3.04 & 1.08 & 2.23 & 1.23 & -6.46 & 377 & .000 & .31 \\
\hline Búsqueda de la información con fin académico & 4.70 & 1.56 & 3.13 & 1.80 & -8.50 & 377 & .000 & .40 \\
\hline
\end{tabular}

Finalmente, en la tabla 5 se observa que existen diferencias significativas en cuanto a tener Internet en casa por parte de los padres de familia y su percepción ante el uso que sus hijos como estudiantes tienen de las TIC. La potencia estadística de estas diferencias es mayor puesto que cumple con los criterios mínimos para determinar que esa diferencia entre las medias se establezca y se sustenta mediante la prueba de Cohen's d.

Tabla 5: Resultados de la prueba t de Student contar con la Internet en casa.

\begin{tabular}{|l|c|l|c|c|c|c|c|c|}
\hline \multicolumn{1}{|c|}{ Dimensiones } & \multicolumn{2}{|c|}{$\begin{array}{c}\text { Si } \\
(n=213)\end{array}$} & \multicolumn{2}{c|}{$\begin{array}{c}\text { No } \\
(n=165)\end{array}$} & \multicolumn{1}{c|}{$t$} & $g l$ & $p$ & $d$ \\
\hline & $\mathrm{M}$ & $\mathrm{DS}$ & $\mathrm{M}$ & $\mathrm{DS}$ & & & & \\
\hline Realización de actividades académicas & 2.77 & 1.13 & 2.00 & 1.05 & -6.37 & 377 & .000 & .31 \\
\hline Socialización & 4.65 & 1.86 & 3.03 & 1.90 & -8.33 & 377 & .000 & .39 \\
\hline Actividades recreativas & 3.11 & 1.10 & 2.38 & 1.17 & -6.21 & 377 & .000 & .30 \\
\hline Búsqueda de la información con fin académico & 4.92 & 1.47 & 3.38 & 1.72 & -9.48 & 377 & .000 & .43 \\
\hline
\end{tabular}

De los resultados expuestos en este estudio, se observó que la percepción de los padres es positiva en cuanto al uso de las TIC en la educación de sus hijos, ya que consideran el uso de la computadora y la Internet como un apoyo para el aprendizaje de sus hijos en casa. Esto se relaciona con los resultados observados por Solís y Aguiar (2017) quienes identificaron a través de la percepción de los padres y madres de familia que sí llevan a cabo tareas de participación con sus hijos (aprendizaje en casa); por lo tanto (Aguilar y Leiva (2012) aseguran que es más fácil trabajar y aprender juntos, así como movilizar recursos de participación efectiva en el contexto escolar si se utilizan de manera adecuada los canales virtuales de comunicación; asimismo agregan que esto (la comunicación) puede ayudar a superar la incompatibilidad horaria y las distintas problemáticas familiares, en la que influye la carencia de una verdadera compatibilidad de vida familiar y laboral que, a veces, convergen en una demanda de más horas de permanencia de los menores en la escuela, mientras que ellos desean una mayor presencia de los padres y madres en el hogar. Del mismo modo, Valdés et al., (2009) encontraron en un estudio sobre la participación de los padres de alumnos de educación primaria en las actividades académicas de sus hijos, que su labor en cuanto al apoyo educativo a sus hijos se realiza en el contexto familiar.

Con respecto a los diversos factores que se analizaron en esta investigación como fueron Realización de actividades académicas, Socialización, Actividades recreativas y Búsqueda de la información para fines académicos, se demostró que existe correlaciones significativas entre los factores que se encargan de medir la percepción del padre de familia sobre el uso que tienen sus hijos de las TIC en las dimensiones expuestas. A pesar de lo anterior, en un estudio sobre la comunicación en la familia a través de las TIC (desde la percepción de los adolescentes) Hernández et al., (2014) observaron que el uso desmedido de las TIC por parte de los jóvenes ha provocado que éstos se comuniquen con más frecuencia con sus similares que con sus padres contribuyendo en un menor apoyo en las actividades escolares; esto podría tener su causa en la mediación parental restrictiva que regula de una manera u otra el comportamiento de los adolescentes, limitando la interacción por medios electrónicos con la familia (Rodríguez de Dios et al., 2018).

Para conocer si existen diferencias significativas entre el estado civil de los padres de familia y las dimensiones del instrumento aplicado (variables sociodemográficas), los resultados mostraron que no existen diferencias significativas en cuanto al estado civil de los padres de familia y su percepción ante el uso que sus hijos como estudiantes tienen de las TIC. Aunque no precisaron datos de esta variable en su estudio Parent's perception over use of ICT in education, Baytak et al. (2012) observaron que independientemente de la edad y estado civil de los padres de familia, éstos se deben capacitar para guiar y ayudar a sus hijos adolescentes en uso 
de las TIC en el aprendizaje. Por otro lado, se observó que existen diferencias significativas en cuanto a tener conexión a la Internet y computadora en casa por parte de los padres de familia y su percepción ante el uso que sus hijos como estudiantes tienen de las TIC. No obstante, durante el proceso de formación del estudiante, los padres de familia deben tener una participación activa supervisando y monitoreando en uso de las TIC dentro del hogar (Urías, Angulo y Torres, 2015).

Así pues, lo anterior permitirá a las autoridades, investigadores y participantes en el estudio: (1) ofrecer información a los padres con el fin de que conozcan las ventajas que ofrecen las TIC en el aprendizaje de sus hijos que estudian el nivel secundaria; (2) ofrecer capacitación para que los padres aprendan a orientar a sus hijos sobre cómo usar las TIC con fines académicos y supervisar sus actividades recreativas; (3) invitar a los padres de familia a participar en conferencias, reuniones o talleres donde se desarrollen diferentes estrategias para el uso correcto de las TIC; (4) ampliar la muestra y replicar el estudio en otros contextos similares; y (5) no generalizar los resultados aquí expuestos a otros padres de familia debido a que los resultados observados fueron desde una perspectiva personal a una muestra representativa en un contexto específico.

\section{CONCLUSIONES}

De acuerdo a los resultados del estudio, de su discusión y análisis, se pueden extraer las siguientes conclusiones:

1. Existe percepción positiva de los padres de familia hacia el uso de las TIC en la educación de sus hijos.

2. Los padres de familia consideran fundamental en la educación de sus hijos la utilización de las TIC.

3. Existe una alta frecuencia donde los padres de familia reportan utilizar las TIC en casa para apoyar el proceso formativo de sus hijos; $y$

4. La mayoría de padres de familia reportan tener computadora e Internet en casa para contribuir a la formación de los hijos.

\section{AGRADECIMIENTOS}

Al Instituto Tecnológico de Sonora (ITSON) de México por proporcionarnos los recursos humanos (docentes, alumnos e investigadores), la infraestructura tecnológica (laboratorios de cómputo, equipo y software) y los recursos financieros (apoyo a la investigación) a través del Programa de Fomento a la Investigación (PROFAPI); el Cuerpo de Académico de Tecnología Educativa en la Sociedad del Conocimiento (ITSON-CA27); y el Programa Fortalecimiento de la Calidad Educativa (PFCE - 2019).

\section{REFERENCIAS}

Aguilar, M. y J. Leiva, La Participación de las Familias en las Escuelas TIC: Análisis y Reflexiones Educativas, 1133-8482, Pixel-Bit. Revista de Medios y Educación, (40), 7-19 (2012)

Angulo, A. J., L. Márquez y otros tres autores, Escuela y familia ante la integración de las TIC en el aprendizaje; En Educación y salud. Evidencias y propuestas de investigación en Sonora, 1ª Ed., por García, F. R., Mortis, L. V., Tánori, Q. J. y Sotelo Q. T., pp. 159 - 167, Editorial Fontamara: México (2015)

Asociación Mexicana de Internet, 15etudio sobre los hábitos de los usuarios de Internet en México 2019 (2019)

Baytak, A., C. Akbiyik y M. Usak, Parents' Perception over Use of ICT in Education, Technics Technologies Education Management, 7(3), 1158 - 1167 (2012)

Carranza, A. M., C. Islas y otros tres autores, "The use of ICT by high school students and the impact on academic performance". 11 ${ }^{\text {th }}$ International Technology, Education and Development Conference, Valencia, España, Marzo (2017)

Cunha, J., P. Rosário y otros cinco autores, Parents' Conceptions of their Homework Involvement in Elementary School, ISSN: 0214-9915, Psicothema, 27(2), 159-165 (2015)

Epstein, J. L., "School, family, and community partnerships: Preparing educators and improving schools", 2a ed., 620, Press, Philadelphia, PA (2011)

Epstein, J. L. y M. Sanders y otros cuatro autores, "School, family, and Community Partnerships", $2^{\mathrm{a}}$ Ed., 430, A Sage Publications Company, United States of America (2002)

Epstein, J. y S. Sheldon, Moving Forward: "Ideas for research on school, family, and community partnerships, En Handbook for Research in Education, $1^{\text {a }}$ Ed., Engaging ideas and enriching inquiry por C. Chifton y S. Ronald, 1734, Sage Publications, Thousand Oaks (2006)

Fernandez-Batanero, J. y M. Colmenero-Ruiz, Ict and Inclusive Education: Attitudes of the Teachers in Secondary Education, doi: http://dx.doi.org/10.3926/jotse.208, Journal of Technology and Science Education, 6 (1), 19-25 (2016)

García Ch. M. y L. Rey, Teachers' Beliefs and the Integration of Technology in the EFL Class, ISSN: 0120-5927, HOW a Colombian Journal for Teachers of English, 20(1), 51-72, (2013) 
Gurpegui, C., Nuevas tecnologías en la familia, Gobierno de Aragón, España (2010)

Hernández, P. M., P. López y Sh. Sánchez, La Comunicación en la Familia través de las TIC. Percepción de los Adolescentes, ISSN: 1577 - 0338, Pulso, 37, pp. 35 - 38 (2014)

Krajka, J. y M. Kleban, E-training in Practical Teacher Development from Local to Global Connections. International Journal of Continuing Engineering Education and Life-Long Learning, 24(1), 96-106 (2014)

Kumar, S. y B. Ben, Integration of Learning Technologies into Teaching within Fijian Polytechnic Institutions, International Journal of Educational Technology in Higher Education, 13 (1), 1-17 (2016)

Kwok, D. y S. Yang, Evaluating the Intention to use ICT Collaborative Tools in a Social Constructivist Environment. International Journal of Educational Technology in Higher Education, 14 (1), 1-14 (2017).

Lewis, A, Helping Young Urban Parents Educate Themselves and their Children, ISSN-0889-8049, 2-12, Digests, (1992)

Losada, D., I. Karrera y E. Jiménez de Aberasturi, Factors Facilitating Successful Educational Innovation with ICT in Schools, ISSN: 1136 - 1034, Revista de Psicodidáctica, 17 (1), 113-134 (2012)

Mantilla, C. M., M. Cedillo y J. Valenzuela, "Competencias en TIC, desarrolladas por estudiantes de secundaria, de acuerdo a los Estándares de la Sociedad Internacional para la Tecnología en la Educación”. Congreso Iberoamericano de Ciencia, Tecnología, Innovación y Educación, 27, Buenos Aires, Argentina (2014)

Martiniello, M., Participación de los padres en la Educación: Hacia una Taxonomía para América Latina. Central American Project, (1999)

Matías, D. A., P. Silva y J. Viana, Children's use of ICT, Family Mediation, and Social Inequalities, Issues in Educational Research, 28 (1), 6176 (2018)

Morales L. Y., Arguments for the Definition of the Ict Skills for Prospective Teachers: University Student Perception about the role of Teachers of Mathematics in Secondary, ISSN: 1516-7313, Ciência \& Educação (Bauru), 17 (3), 757-769, (2011)

Mutch, C. y S. Collins, Partners in Learning: Schools' Engagement With Parents, Families, and Communities In New Zealand, ISSN: 1059 - 308X, School Community Journal, 22(1) (2012)

Noguera F. I, How Millennials are Changing the Way we Learn: the State of the Art of Ict Integration in Education, ISSN: 1138-2783 , RIED. Revista Iberoamericana de Educación a Distancia, 18(1), 45-65 (2015)

Perales, F. y M. Escobedo, La Participación social en la Educación: entre Propuestas Innovadoras y Tradición Educativa, ISSN: 1607-4041, Revista electrónica de investigación educativa, 18(1), 68 - 81 (2016)

Rodríguez de Dios, I., J. Van y J. Igartua, A Study of the Relationship Between Parental Mediation and Adolescents' Digital Skills, Online Risks and Online Opportunities, Computer and Human Behavior, 82, 186 - 198 (2018)

Solís C. F. y R. Aguiar, Análisis del Papel del Involucramiento de la Familia en la Escuela Secundaria y su Repercusión en el Rendimiento Académico, ISSN: 2007-7033, Sinéctica, (49), (2017)

Urías, M. M., J. Angulo y C. Torres, Parent Perception towards ICT in Secondary Education. Conferencia internacional ICAICTSEE, 82 - 86, Sofía, Bulgaria, 13 - 14 de noviembre (2015)

Urías, M. M., J. Angulo, A. Valdés, C. A. y C. Torres, Propiedades Psicométricas para Medir la Percepción de Padres de Familia sobre sus Hijos Estudiantes ante el Uso de las TIC. Educación y Salud. Evidencias y propuestas de investigación en Sonora, 1ํㅡㄹ Ed., por García, F. R., Mortis, L. S., Tánori, Q. J., y Sotelo, Q. T., 207218, Fontamara, México (2015)

Valdés C. A. y M. Urías, Creencias de Padres y Madres acerca de la Participación en la Educación de sus Hijos, ISSN: 2428-6167, Perfiles educativos, 33(134), 99-114 (2011)

Valdés, Á. A., M. Martín y P. Sánchez, Participación de los Padres de Alumnos de Educación Primaria en las Actividades Académicas de sus Hijos, ISSN: 1607-4041, Revista Electrónica de Investigación Educativa, 11(1), 1 - 17 (2009) 